\title{
Functional glutamate transport in optic nerve axons and glia.
}

Amaia Arranz ${ }^{2}$, Ali Hussein ${ }^{1}$, James Alix ${ }^{1}$, Fernando Pérez-Cerdá ${ }^{2}$, Natalie Allcock ${ }^{1}$, Carlos Matute $^{2}$ and Bob Fern ${ }^{1}$

${ }^{1}$ Department of Cell Physiology and Pharmacology, University of Leicester, Leicester, England. ${ }^{2}$ Department of Neurosciences, Universidad del País Vasco, 48940-Leioa, Spain

Short Title: Glutamate transporters in glia and axons

Associate Editor:

Corresponding Author:

Dr Bob Fern

Department of Cell Physiology \& Pharmacology, University of Leicester, P.O.

Box 138, University Road, Leicester, LE1 9HN, England.

Telephone: $+44(0) 1162523098$

Fax: $+44(0) 1162525045$

This work was supported by the National Institutes of Neurological Disorders and Stroke grant NS 44875 to R.F.

Key words: Astrocyte; Axon; Glia; Glutamate transport; Oligodendrocyte; White matter 


\begin{abstract}
Glutamate uptake and potential release via $\mathrm{Na}^{+}$-dependent glutamate transporters is crucial to CNS function and to various forms of injury. Evidence for glutamatemediated damage of oligodendroglia somata and processes in white matter suggests that glutamate regulation in white matter is of particular clinical importance. The expression of glutamate transporters was examined in developing mouse and mature mouse and rat white matter using immuno-histochemistry and immuno-electron microscopy. EAAC1 was the major glutamate transporter detected in oligodendroglia cell membranes in both neonatal and mature optic nerve while GLT1 was the most heavily expressed transporter in the membranes of astrocytes. Both EAAC1 and GLAST were also seen in adult astrocytes but there was little membrane expression of either in the neonate. GLAST, EAAC1 and GLT1 were expressed in neonatal axons with significant amount of GLT1 present in the axolemma, while in mature axons EAAC1 was abundant at the node of Ranvier. Functional glutamate transport was probed in developing mouse optic nerve revealing $\mathrm{Na}^{+}$-dependent, TBOA-blockable uptake of D-aspartate in astrocytes, axons and oligodendrocytes. The data show that in addition to oligodendroglia and astrocytes, axons represent a significant potential sink and source for extracellular glutamate in white matter.
\end{abstract}

\title{
Introduction
}

Glutamate is the major excitatory neurotransmitter in the mammalian CNS but is also neurotoxic and has been implicated in important neurological diseases. High-affinity, $\mathrm{Na}^{+}$-dependent glutamate transporters are responsible for clearing synaptically released glutamate from the extracellular space of the CNS (Gegelashvili and Schousboe, 1998; Anderson and Swanson, 2000; Danbolt, 2001). Of the five glutamate transporters that have been cloned, three are widely distributed in the mature CNS (Rothstein et al., 1994; Furuta et al., 1997a; Gegelashvili and Schousboe, 1998; Danbolt, 2001). GLAST and GLT-1 are commonly known as glial glutamate transporters, whereas EAAC1 is regarded as the principal transporter in neurons. The voltage and $\mathrm{Na}^{+}$-dependence of these 
glutamate transporters can result in the reversal of transport under ischemic conditions, which can act as one source of the extracellular glutamate that is responsible for excitotoxic cell injury during disorders such as stroke (Attwell et al., 1993; Anderson and Swanson, 2000). In addition to injury in grey matter regions of the brain, stroke affects white matter regions that are made up of axons, astrocytes and oligodendrocytes (Stys, 2004). Excitotoxic injury has also been implicated in other forms of white matter disease such as spinal cord injury and multiple sclerosis (Li et al., 1999; Matute et al., 2001). Oligodendrocyte damage is central to loss of function in ischemic white matter injury and is thought to result from glutamate-mediated excitotoxicity (Pantoni et al., 1996; Tekkok and Goldberg, 2001; Dewar et al., 2003).

In addition to adult disease, white matter injury is a particularly important component of the lesions that underlie neuro-developmental disorders such as cerebral palsy, which in many cases appear to be ischemic in origin (Kinney and Back, 1998; Volpe, 2001, 2003). Ischemia evokes significant rises in extracellular glutamate concentration in white matter (Shimada et al., 1993; Chiu and Kriegler, 1994), and recent immuno-electron microscopy studies suggest that glutamate is released from both astrocytes and axons in ischemic neonatal white matter ( $\mathrm{Li}$ et al., 1999; Wilke et al., 2004). There is little information about the type of transporters mediating glutamate uptake in white matter tracts of the central nervous system. Prior studies have shown EAAC1, GLAST and GLT-1 expression in mature white matter (Choi and Chiu, 1997; Furuta et al., 1997a; Domercq and Matute, 1999; Kugler and Beyer, 2003), although the cellular distribution of these transporters is controversial, and little is known regarding their cellular distribution in neonatal white matter. Several studies have shown GLT-1 expression in neonatal central axons using light microscopy (Kugler and Beyer 2003; Furuta et al., 1997a), but the close apposition of axonal and glial membrane casts the axonal expression of this "glial" transporter into doubt. However, membrane expression of GLT-1 in embryonic spinal cord axons before the appearance of astrocytes has been shown using immuno-electron microscopy (Yamada et al., 1998). Recent studies have shown that interruption of glutamate transport in white matter leads to excitotoxic injury of axons and glia (Domercq et al., 2005), highlighting the importance of glutamate clearance in these structures. 
Here we use transgenic mice where EGFP expression is under the control of glial cell type specific promoters, coupled with standard and electron immuno-histochemistry to examine glutamate transporter expression in the neonatal and mature optic nerve. Staining for the uptake of exogenously perfused D-aspartate and for the localization of glutamate was used to confirm that transporter expression was functional, revealing high levels of transporter expression in glia and in particular within axons.

\section{Materials and Methods}

Transgenic mice $(\mathrm{FVB} / \mathrm{N})$ carrying the EGFP coding sequence under the control of CNP promoters 1 and 2 (Yuan et al., 2002) were kindly donated by the laboratory of Vittorio Gallo (CNMC Research, Washington DC). Heterozygous males were mated with wild type females and transgenic littermates identified. A tendency for epileptic seizures in the colony was largely removed by out-breading with wild-type animals and subsequent back-crossing. Transgenic mice (FVB/N) with EGFP under control of the GFAP promoter were obtained from The Jackson Laboratory (Bar Harbour, Maine). All animals were maintained in accordance with local ethical guidelines.

For immuno-histochemistry, optic nerves were dissected in 0.1M PBS and fixed in 4\% parafomaldahyde for $30 \mathrm{~min}$. Nerves from P14-17 mice are termed "neonatal" and from $>$ P30 as "adult" throughout. The optic nerves were subsequently incubated in $0.1 \mathrm{M}$ PBS plus $20 \%$ sucrose $\mathrm{w} / \mathrm{v}$ for $5 \mathrm{~min}$ prior to freeze-sectioning and subsequent blocking for $60 \mathrm{~min}$ in $0.1 \mathrm{M}$ PBS $10 \%$ fetal goat serum $+0.5 \%$ Triton-X 100 and incubated in this solution plus primary antibody at $4^{\circ} \mathrm{C}$ overnight. Affinity-purified rabbit polyclonal antibodies against GLT1 (EAAT2), GLAST (EAAT1) and EAAC1 (EAAT3) together with specific blocking peptides were obtained from Alpha Diagnostics (San Antonio, TX, USA) and were used at a 1:100 dilution. Mouse monoclonal antibodies against neurofilament-70 (NF-70, 1:200) were obtained from Chemicon Europe (Southampton, UK). Affinity-purified rabbit polyclonal antibody raised against D-aspartate (1:2000) was obtained from Cell Sciences (Canton, MA) and rabbit polyclonal anti CNPase was obtained from Sigma (1:100). The appropriate Alexa-conjugated secondary antibody (Cambridge Bioscience, UK) was applied for 120 min following washing and single-plan fluorescent sections were imaged at x60 on an Olympus scanning confocal microscope. 
Alexafluor-568 conjugated secondary antibodies were employed when labelling transgenic mice to allow spectra discrimination between the label and EGFP (emission maxima at $520 \mathrm{~nm}$ ).

For D-aspartate uptake studies, optic nerves were dissected and placed in artificial cerebrospinal fluid (aCSF), composition (in $\mathrm{mM}$ ): $\mathrm{NaCl}, 126 ; \mathrm{KCl}, 3 ; \mathrm{NaH}_{2} \mathrm{PO}_{4}, 2$; $\mathrm{MgSO}_{4}, 2 ; \mathrm{CaCl}_{2}, 2 ; \mathrm{NaHCO}_{3}, 26$; glucose, $10 ; \mathrm{pH}, 7.45$, bubbled with 5\% $\mathrm{CO}_{2} / 95 \% \mathrm{O}_{2}$ and maintained at $37^{\circ} \mathrm{C}$. This solution contained $500 \mu \mathrm{M}$ D-aspartate and trials indicated that a 60 min incubation period produced good cell loading. Shorter period of incubation failed to produce reliable D-aspartate staining and the relatively prolonged incubation period presumably reflects the slow penetration of D-aspartate into a whole-mount preparation. Following the incubation, optic nerves were washed for $5 \mathrm{~min}$ in aCSF prior to processing for immuno-histochemistry (see above). In addition to parafomaldehyde, glutaraldehyde $(3 \%)$ was tried as a fixative but failed to yield useful staining. Zero- $\mathrm{Na}^{+}$ aCSF had $\mathrm{NaCl}$ replaced with $\mathrm{N}$-methyl-D-glucamine (NMDG), $\mathrm{NaH}_{2} \mathrm{PO}_{4}$ replaced with $\mathrm{KH}_{2} \mathrm{PO}_{4}$ and $\mathrm{NaHCO}_{3}$ replaced with choline- $\mathrm{HCO}_{3}$. All chemicals were obtained from Sigma (UK).

For pre-embedded glutamate transporter electron microscopic immunohistochemistry in adult white matter we used Sprague-Dawley rats. Animals were deeply anesthetized by intraperitoneal injection of $20 \%$ cloral hydrate in distilled water and were transcardially perfused with $500 \mathrm{ml}$ of fixative containing 4\% paraformaldehyde, $0.1 \%$ glutaraldehyde and $0.2 \%$ picric acid in $0.1 \mathrm{M}$ phosphate buffer ( $\mathrm{PB}, \mathrm{pH} 7.4)$. Perfusates were used at room temperature (RT). After perfusion, optic nerves were removed and post-fixed in $0.1 \mathrm{M} \mathrm{PB}$ for about $3 \mathrm{~h}$. Both longitudinal and transversal vibratome sections (60-70 $\mu \mathrm{m}$ thick) were collected in 0.1 M PB and washed several times in phosphate-buffered saline (PBS, $\mathrm{pH}$ 7.4). Floating sections were preincubated in $10 \%$ goat serum in PBS for $1 \mathrm{~h}$ at RT. Sections were then incubated with the anti-GLAST (5 $\mu \mathrm{g} / \mathrm{ml}$; (Danbolt et al., 1992)), anti-GLT1 (0.4 $\mu \mathrm{g} / \mathrm{ml}$; (Rothstein et al., 1994)) and anti-EAAC1 $(1.14 \mu \mathrm{g} / \mathrm{ml}$; (Rothstein et al., 1994)) antisera diluted in blocking solution, for 3 days at $4^{\circ} \mathrm{C}$ and continuous shaking. In control sections the primary antibody was omitted. After several washes in PBS for about $2 \mathrm{~h}$, tissue sections were incubated in 1.4 nm gold-labelled goat anti-rabbit IgG (Fab' fragment, 1:100 in blocking solution; 
Nanoprobes Inc.) for $4 \mathrm{~h}$ at RT. Thereafter, the sections were washed in PBS overnight at $4^{\circ} \mathrm{C}$ and fixed in $1 \%$ glutaraldehyde for $10 \mathrm{~min}$. Following washes in MQ water, gold particles were silver-intensified with an HQ Silver Enhancement kit (Nanoprobes Inc.) for about $10 \mathrm{~min}$. After several washes in MQ water, the gold-labeled, silver-intensified tissue sections were fixed in $0.1 \mathrm{M}$ PB for $30 \mathrm{~min}$, followed by $1 \%$ osmiun tetroxide in 0.1 M PB and fixed again in 0.1 M PB for $30 \mathrm{~min}$. Sections were then dehydrated and embedded in Epon. Ultrathin sections were collected on mesh nickel grids, stained with $1 \%$ uranyl acetate and 1\% lead citrate both in MQ water and examined using a PHILIPS EM208S electron microscope. To estimate the density of silver-intensified particles, we randomly took 15 micrographs at 16-20 k magnification which were analysed with Scion Image software (NIH, Frederick, MD). Omission of primary antibodies in control sections resulted in the absence of immunolabelling.

For post-embedded glutamate, glutamate transporter and D-aspartate electron microscopic immuno-histochemistry optic nerves from P10 mice were post-fixed in 3\% gluteraldehyde/Sorenson's buffer. Nerves were post-fixed with $2 \%$ osmium tetroxide and dehydrated prior to infiltration in epoxy. Sections were counterstained with uranyl acetate and lead citrate and examined with a Jeol 100CX electron microscope, see (Thomas et al., 2004) for further details. Antibodies were the same as listed above for light immunohistochemistry.

\section{Results}

EAAC1, GLAST and GLT-1 were all detected in wild-type neonatal optic nerve by antibody staining (Fig 1, left panels). In each case the staining was ablated by preincubation with a specific blocking peptide (Fig 1, right panels). EAAC1, GLAST and GLT-1 staining was apparent in cell bodies throughout the nerve (arrows). Staining of somata will correspond to glial cell bodies in this neuron-free preparation. Expression of all three proteins were not restricted to somata and a significant level of staining was seen throughout the nerve presumably due to staining of glial processes and/or axons. Such staining was at a somewhat lower level of intensity to that seen in the somata but occupied a greater proportion of the nerve and may not have been noted in previous studies due to image thresholding. In the current investigation, we initially imaged 
control sections to determine the imaging parameters required to eliminate background fluorescence prior to using the same parameters for tests sections, thus ensuring that our images were not biased towards bright objects at the expense of dim objects.

Functional high affinity $\mathrm{Na}^{+}$-dependent glutamate transporters will transport exogenous D-aspartate into cells which can subsequently be assessed by antibody staining (Gundersen et al., 1993; Gundersen et al., 1995). Perfusion with $500 \mu$ M Daspartate for $60 \mathrm{~min}$ produced extensive D-aspartate staining in somata and non-somatal regions of the neonatal optic nerve (Fig 2 A). Pre-absorbing the D-aspartate antibody for $30 \mathrm{~min}$ in $500 \mu \mathrm{M}$ D-aspartate prior to staining ablated the immuno-staining (Fig $2 \mathrm{~B}$ ). Daspartate uptake was largely abolished by zero- $\mathrm{Na}^{+}$conditions (Fig $2 \mathrm{C}$ ) and by the specific transport inhibitor DL-threo-beta-benzyloxyaspartate (TBOA, $10 \mu \mathrm{M}$; Fig $2 \mathrm{D})$, confirming the expression of functional high affinity $\mathrm{Na}^{+}$-dependent glutamate transport in somata and non-somatal regions of the optic nerve. The period of D-aspartate exposure was determined empirically by exposing nerves for a range of times and assessing uptake, 60 min exposure producing significant D-aspartate loading into the nerves.

Figure 1 and 2 near here

The expression pattern of GLT-1, GLAST and EAAC1 and the pattern of uptake of D-aspartate into areas surrounding somata may indicate a significant axonal presence of glutamate transporters. Double labelling for neurofilament revealed clear colocalization of this axonal marker with GLT-1, EAAC1 and GLAST (Fig 3 A-C). Immuno-gold labelling for EAAC1 revealed that while this transporter is expressed within oligodendrocyte processes that are closely apposed to axons, and to a limited extent within the axoplasm, no staining was apparent within the axolemma itself (Fig 3 D, E).Post-imbedded immuno-staining for GLAST was not successful, but immuno-gold GLT-1 labelling revealed extensive expression within the axolemma both of premyelinated and myelinated axons, in addition to staining of near-bye astrocyte processes (Fig 3 F, G).

Figure 3 near here 
Light microscopy revealed D-aspartate uptake into axons within in pre-incubated nerves (Fig 4 A, B, arrows), in addition to uptake into glial processes running parallel to axons (Fig 4 C, D, arrows). Although axonal D-aspartate uptake was somewhat punctate in many regions, areas of diffuse staining along the centre of axons were apparent and presumably represent axoplasmic accumulation. At the ultrastructural level, postembedded immuno-gold labelling for D-aspartate in pre-incubated nerves revealed significant loading into axons and oligodendrocytes, with highly variable loading into astrocytes (Fig 4, E). Staining for glutamate in control nerves revealed the presence of the neurotransmitter in astrocytes, axons and oligodendrocytes (Fig 4 F). Post-embedded staining was used in the neonatal tissue since in our hands it allows a greater level of tissue detail to be observed, which is required to examine sub-cellular expression in small diameter neonatal axons.

Figure 4 near here

Blinded counting of gold particles showed that astrocytes accumulate significantly more D-aspartate than either axons or oligodendrocytes within the optic nerve, although between them the two elements of myelinated axons have a greater capacity for D-aspartate uptake than do astrocytes (Fig $5 \mathrm{~A}$ ). The population distribution of gold-particle density within astrocytes was found to be highly variable, with a population of astrocytes that accumulated little D-aspartate and other cells that accumulated mush more (Fig 5 B).

Figure 5 near here

Transgenic mice in which EGFP expression is under the control of glial cell specific promoters were used to probe the expression of glutamate transporters and the uptake of D-aspartate into astrocytes and oligodendrocytes. Optic nerves from neonatal EGFP-GFAP mice retained EGFP fluorescence following fixation. Immuno-staining revealing an absence of EAAC1, a relatively high level of GLT-1 expression in these 
cells (Fig 6 A, B). Only diffuse staining for GLAST was found in glial cells in neonatal mouse optic nerve, which could not be discriminated from background levels of staining (not shown). At the ultrastructural level, gold particles were present at high density in the cell membranes of astrocyte processes (Fig 6 C, arrows). Assessed at the light level, the capacity of astrocytes to take up exogenous D-aspartate was found to be highly variable with some cells having undetectable levels of D-aspartate and other having very high levels (Fig 6 D, E).

Figure 6 near here

At the light level, the majority of oligodendrocytes in neonatal EGFP-CNP optic nerve expressed EAAC1, with only diffuse low levels of staining observed for GLT-1 (Fig 7 A-D). A population of EAAC1 (-) oligodendrocytes were also observed (not shown). D-aspartate uptake into oligodendrocytes was at a somewhat higher level than that seen in surrounding tissue (Fig 7 E-G) and D-aspartate (-) cells were not seen. Uptake was often particularly marked within processes of EGFP-CNP oligodendrocytes (Fig $7 \mathrm{G}$ ), but the EGFP fluorescence was retained only poorly in the more distal processes that are beginning to ensheath axons at this point in development. In separate experiments oligodendrocytes in pre-loaded wild-type mice were antibody stained against CNPase to show these fine processes in greater detail, revealing significant D-aspartate uptake into these structures (Fig 7 E, F arrows). This also revealed CNPase (-) axon-like structures loaded with D-aspartate (Fig 7 E, F arrow heads), which correspond to the NF$70(+)$ axons seen in Figure 4.

Figure 7 near here

Glutamate transporter expression was also examined in mature mouse and rat optic nerve, revealing a similar pattern in both (we will show the data from the rat). Mature optic nerve astrocytes were found to mainly expressed GLT-1 in their somata and processes (Fig $8 \mathrm{~A}$ ), in agreement with the idea that this is the major glutamate transporter present in these cells. GLT-1 -particles are almost exclusively found in plasma membranes within astroglia. As in neonatal optic nerves, GLT-1 was also 
expression in oligodendrocytes but in the adult cells prortein was found in th excell membranes (Fig 8 B). No GLT-1 expression was found in axonal tracts of the adult optic nerve (Fig $8 \mathrm{C}, \mathrm{D})$.

Figure 8 near here

GLAST was also expressed in both astroglial somata and processes of the adult optic nerve (Fig 9 A, C), but the density of immunoparticles was lower than for GLT-1 (Fig 11 A). As for GLT-1, GLAST was mostly associated to plasma membranes but there were some GLAST immuno-labelling in the cytoplasm of astroglia. GLAST was also found in oligodendrocytes, especially in their myelin sheaths (Fig 9 B). GLAST was not detected in axonal tracts and at the nodes of Ranvier (Fig 9 C, D).

Figure 9 near here

EAAC1-immunolabelling in the adult optic nerve was mainly present in axonal tracts and it was particularly abundant at nodes of Ranvier (Fig 10 C, D). In addition, most oligodendrocytes and astrocytes expressed EAAC1 (Fig 9 A, B). Curiously, EAAC1 immuno-particles were often present in cytoplasm, a feature which could be due to the rapid trafficking and recycling of this transporter between the cytoplasm to the plasma membrane (Danbolt, 2001).

Figure 10 near here

A quantitative analysis of the distribution of glutamate transporters revealed that GLT-1, GLAST and EAAC1 are all expressed at high levels in astrocytes, whereas GLT1 and EAAC1 were the only types present in oligodendrocyte somata and their expression in these cells was lower (Fig 10). In turn, GLAST was also observed in myelin sheaths. Finally, EAAC1 was found at high levels in axons, particularly at the nodes of Ranvier. 
Figure 11 near here

\section{Discussion}

We have shown a high capacity for Na-dependent, TBOA-blockable, D-aspartate uptake into central white matter astrocytes, axons and oligodendrocytes in two rodent species at two developmental points. All three of these cellular compartments also contain cytoplasmic glutamate. These findings indicating that glutamate regulation is important in white matter and that it is achieved by glutamate transporter expression in axons and oligodendroglia in addition to astrocytes. Glutamate transporters were shown to be expressed in white matter oligodendrocytes, axons and astrocytes both during development and in the adult. This implies that the extracellular concentration of glutamate is carefully regulated by these proteins in white matter just as it is in grey matter.

White matter glutamate transporter expression has previously been examined mainly in the adult rat optic nerve, a preparation that contains protein and RNA for GLT1, EAAC1 and GLAST (Choi and Chiu, 1997; Domercq et al., 1999). Two groups have published different cell distributions of these transporters in this preparation. Domercq et al (1999) reported GLAST expression in interfascicular oligodendrocytes, EAAC1 in a population of unidentified glia and GLT-1 in astrocytes. In contrast, Kugler and Beyer (2003) found GLAST expression in most astrocytes, a population of EAAC1 $(+)$ astrocytes and GLT-1 expression in oligodendrocytes and axons. Kugler and Beyer (2003) used $\beta$-tubulin as an oligodendrocyte marker, which may also be expressed in astrocytes (e.g., Medrano and Steward, 2001), and it is possible that some cells identified in their study as oligodendrocytes were in fact astrocytes. In addition to optic nerve, high levels of GLAST, GLT-1 and EAAC1 expression have been reported in other white matter structures such as sub-cortical white matter, in particular during post-natal development (Furuta et al., 1997a).

The current study used pre-embedded immuno-electron microscopy to demonstrate GLT-1, GLAST and EAAC1 expression within the cell membrane of mature astrocytes and EAAC1 expression within the cell membrane of oligodendrocytes. There has been no previous examination of the cellular distribution of glutamate transporter 
expression in neonatal white matter. The current study employed EGFP expression linked to cell-specific promoters to identify glial cell types in neonatal mouse white matter, removing any possibility of antibody interactions during double labelling, and used postembedded immuno-electron microscopy to examine the sub-cellular distribution of the proteins. In the neonate, membrane expression of GLT-1 was found in astrocyte processes, with only cytoplasmic expression seen in oligodendrocytes. EAAC1 was expressed in oligodendrocytes and not in astrocytes, while staining for glial GLAST expression was not easily discriminated from background levels. The EGFP-expression approach was also used to examine mature mouse optic nerve, producing results similar to those described with pre-embedded immuno-electron microscopy. Uptake of exogenous D-aspartate was also employed to prove that developing white matter glial glutamate transporters were functional in situ.

The current study revealed intense membrane expression of GLT-1 in axons in neonate white matter. In contrast, mature axons did not express GLT1 or GLAST while strong EAAC1 expression was found largely localized to the node of Ranvier. Previous studies have shown GLT-1 expression in immature mouse spinal cord axons up to the first post-natal week (Yamada et al., 1998), while EAAC1 is present in mature cerebellar Purkinje cell axons (Furuta et al., 1997b). The high level of D-aspartate uptake and the localization of glutamate within axons confirmed the presence of functional transport, which in terms of D-aspartate uptake capacity was comparable to that seen in glial cells and occupied a larger proportion of the nerve. Axonal uptake was blocked by either $\mathrm{Na}^{+}$ removal or by the specific transport inhibitor TBOA and this is the first direct proof of functional glutamate transport expression in axons. The high level of expression of EAAC1 at the nodes of Ranvier indicates a high level of glutamate regulation in the node in mature axons.

Neonatal oligodendrocytes accumulated high levels of D-aspartate during exogenous perfusion, which is consistent with the elevated levels of glutamate transporter expression in these cells at this point in development, in particular EAAC1. Average Daspartate uptake into astrocytes was higher than into any other cell compartment, although the degree of uptake varied considerably from cell to cell. Recent studies have shown rapid astrocyte swelling leading to necrosis in ischemic neonatal white matter, 
which will liberate astrocyte glutamate into the extracellular space in a glutamate transporter independent fashion (Thomas et al., 2004). In addition, the presence of functional GLT1 demonstrates the potential for transport-mediated glutamate release from these cells. Thomas et al (2004) also demonstrated a high resting intracellular glutamate levels in oligodendrocytes that was elevated following ischemia, suggesting both effective glutamate uptake under normal conditions and the absence of uptake reversal during ischaemia. It is not known how $\left[\mathrm{Na}^{+}\right]_{\mathrm{i}}, \mathrm{pH}_{\mathrm{i}}$ and membrane potential are affected by ischemia in these cells but it would appear that the conditions are not met for significant reversal of glutamate uptake in this preparation (but see Back et al 2006). The current findings suggest that glutamate release from axons via reverse transport is a potentially important factor in excitotoxic (Dewar et al., 2003; Wilke et al., 2004) and non-excitotoxic (Oka et al., 1993) white matter damage during ischemia.

Glutamate receptors are expressed in developing glia, and may regulate cell development and fate (Belachew and Gallo, 2004). Expression of AMPA/kainate receptors is particularly marked in immature cells of the oligodendrocyte lineage (Itoh et al., 2002), which in the neonatal optic nerve are in the process of forming an exquisitely close morphological arrangement with neighbouring axons (Butt and Ransom, 1993). We and others have recently demonstrated the presence of functional NMDA-type glutamate receptors in oligodendrocyte processes (Salter and Fern, 2005; Káradóttir et al., 2005; Micu et al., 2006)). The activation of these receptors is likely to be important in a variety of diseases and must have significant developmental consequences. We have now shown high levels of glutamate transporter expression in both developing axons and glia, which is consistent with a tight maintenance of extracellular glutamate in the zones where axons and glia meet. Conjunctions between developing axons and their ensheathing oligodendrocytes and surrounding astrocytes may therefore be analogous to neuronal synapses where release and clearance of extracellular glutamate is essential for cell-cell interaction. It is also possible that axonal glutamate transporters can mediate physiological glutamate release, especially at nodes of Ranvier. The membrane potential of adult rat optic nerve axons has been estimated at $57 \mathrm{mV}$ (Leppanen and Stys, 1997), and $\left[\mathrm{Na}^{+}\right]_{\mathrm{i}}$ at 20-25 mM (LoPachin and Stys, 1995). The corresponding values in neonatal axons are not known, but a low level of Na-K ATPase activity in developing neurons 
may indicate an even smaller resting membrane potential and higher $\left[\mathrm{Na}^{+}\right]_{\mathrm{i}}$ (Vannucci and Vannucci, 2000). Action potentials passing down axons will temporarily produce depolarization and an elevation in $\left[\mathrm{Na}^{+}\right]$, which may in principle stimulate reversal of uptake and net glutamate release via the nodes of Ranvier onto neighbouring oligodendrocytes and astrocytes (Chiu and Kriegler, 1994). 


\section{Figure legends}

\section{Figure 1}

Glutamate transporter expression in neonatal white matter. EAAC1 (A), GLAST (B) and GLT-1 (C) expression has both a somata pattern of expression (arrows) and nonsomata pattern of expression in neonatal optic nerve. Antibody staining is not seen when the antibodies are pre-incubated in the relevant blocking peptides (right side). Scale bar $=10 \mu \mathrm{m}$.

Figure 2

D-aspartate uptake into neonatal white matter is via functional glutamate transport. A: Antibody staining for D-aspartate showing uptake into somata (arrows) and non-somata areas of the pre-incubated optic nerve. B: Pre-absorbing the antibody with Daspartate prior to incubation blocked D-aspartate staining. C-D: D-aspartate uptake was blocked by removing extracellular $\mathrm{Na}^{+}(\mathrm{C})$ or by TBAO (D), a specific glutamate transport blocker. Scale bar $=10 \mu \mathrm{m}$.

Figure 3

Glutamate transporter expression in neonatal mouse axons. A-C: Axons are costained with NF-70 (left panels, green) and for either; EAAC1 (A, middle panel, red), GLAST (B, middle panel, red) or GLT-1 (C, middle panel, red). The images are overlaid on the panels to the right, showing what appears to be significant co-localization of all three transporters in axons (orange). D, E: Immuno-gold reactivity for EAAC1. D: Gold particles are found throughout the cytoplasm and in particular in the cell membrane of oligodendrocytes (arrows). E: Staining is evident in oligodendrocyte processes (asterisks + arrows) that are closely apposed to axons (Ax). Light staining was found in the axoplasm of axons (arrow head), but not in the axolemma. Note on the right a rare gold particle in the cell membrane of an astrocyte process (ap) aligned next to an axon (arrow). F, G: Immuno-gold reactivity for GLT-1. Note the heavy gold-particle labelling within the axolemma in both panels, in addition to staining within the axoplasm and within the 
membrane of astrocyte processes (ap). Scale bar $=10 \mu \mathrm{m}$ in A-C and $1 \mu \mathrm{m}$ in D-G. The cell types are identified using standard criteria for this preparation (Thomas et al 2005).

Figure 4

Glutamate and D-aspartate staining in neonatal mouse optic nerve. A: NF-70 staining of axons. B: D-aspartate staining of the same section, overlaid to the right. Note the presence of D-aspartate staining within axons (arrows). C: NF-70 staining of axons. FD: D-aspartate staining of the same section, overlaid to the right. Note the D-aspartate staining within a NF-70 (-) glial process running parallel to the NF-70 (+) axons. E: Immuno-gold localization of glutamate reactivity showing gold particles within an oligodendrocyte (Oli), an astrocyte (Ast) and within axons (arrows). The box shows absence of staining when the antibody was pre-incubated with glutamate. F: D-aspartate reactivity in pre-incubated optic nerve. Note the gold particles within an oligodendrocyte and within axons (arrows) and the absence of staining within the astrocyte. Scale bars $=$ $1 \mu \mathrm{m}$.

Figure 5

Blinded counting of D-aspartate gold particles. A: Astrocytes accumulate significantly more D-aspartate than either axons or oligodendrocytes within the optic nerve B: The population distribution of gold-particle density within astrocytes was highly variable, with a population of astrocytes that accumulated little D-aspartate. $* * *=$ $\mathrm{P}>0.001$.

Figure 6

Glutamate transporter expression in neonatal white matter astrocytes. A, B: GFP expression in GFAP-GFP astrocytes (green, left) and labelling for either GLT-1 (A, red, middle). The images are overlaid on the right, showing significant expression of GLT-1 in astrocytes with no co-expression of EAAC1. C: Immuno-gold labelling for GLT-1 showing expression in the membrane of astrocyte processes ("ap") and staining only in the cytoplasm of a neighbouring oligodendrocyte ("Oli"). D: GFP expression in 
astrocytes. E: Co-staining for D-aspartate the same pre-incubated nerve. The images are overlaid below, showing D-aspartate uptake into one of the astrocytes with several Daspartate (-) astrocytes present. Note the presence of D-aspartate (+) somata that are GFP $(-)$, presumably oligodendrocytes. Scale bar $=10 \mu \mathrm{m}$ except in "C" where it is $1 \mu \mathrm{m}$.

Figure 7

Glutamate transporter expression in neonatal white matter oligodendrocytes. A: GFP expression in a CNP-GFP oligodendrocyte showing soma and processes. B: EAAC1 reactivity of the same section. Overlay on the right, showing EAAC1 expression on the soma and processes. C: A single CNP-GFP (+) oligodendrocyte. D: GLT-1 reactivity of the same section. Overlay on the right, showing defuse GLT staining in the cell. E: CNPase staining of a wild-type, D-aspartate pre-incubated, nerve. F: D-aspartate staining of the same section. Overlay below, showing clear D-aspartate staining in the CNPase $(+)$ oligodendrocyte processes (small arrows) as well as within CNPase (-) axon-like structures (arrow heads). G: Three GFP (+) oligodendrocytes in a GFP-CNP nerve. H: Daspartate staining of the same section. Overlay below, showing D-aspartate staining within somata and proximal processes of oligodendrocytes. Scale bars $=10 \mu \mathrm{m}$.

Figure 8

Ultrastructural localization of GLAST in adult rat optic nerve. A, C: Transverse and longitudinal sections showing GLAST localization in astrocyte processes (ap) that surround some axons (ax) free of labelling. Note that immuno-particles are present in plasma membranes as well as in the cytoplasm of astrocytes. B: Expression of GLAST in astrocytes and to a lesser extent in oligodendrocytes. D: Axons (ax) and the nodes of Ranvier (R) are unlabelled. Scale bars $=300 \mathrm{~nm}$.

Figure 9

Ultrastructural localization of GLT-1 in adult rat optic nerve. A: Astrocyte processes (ap) with intense GLT-1 immuno-labelling surrounding a group of axons (ax) all devoid of reactivity. B, C: Transverse and longitudinal sections showing that GLT-1 is present at high levels in astrocyte processes (ap) and to a lesser extent in 
oligodendrocytes (oli). D: No expression of GLT-1 is observed in axons and at the nodes of Ranvier. Scale bars $=300 \mathrm{~nm}$.

Figure 10

Ultrastructural localization of EAAC1 in adult rat optic nerve. A, C: Transverse and longitudinal sections showing EAAC1 expression in both axons (ax) and astrocyte processes (ap). Note that immuno-labelling frequently appears in the cytoplasm. B:

EAAC1 is also found in oligodendrocytes (oli) as well as in astrocyte processes (ap) in their vicinity. D: Note that reactivity is particularly abundant at the nodes of Ranvier. Scale bars $=300 \mathrm{~nm}$.

Figure 11

Quantitative analysis of the distribution of glutamate transporters in adult optic nerve. All transporters are abundantly expressed in astrocytes. Oligodendrocytes express lower levels of GLT-1 and EAAC1, and myelin has GLAST. Axons and Ranvier nodes have moderate and high levels of EAAC1, respectively. $* \mathrm{p}<0.05, * * * \mathrm{p}<0.001$, as compared to control sections. 


\section{References}

Anderson CM, Swanson RA (2000) Astrocyte glutamate transport: review of properties, regulation, and physiological functions. Glia 32:1-14.

Attwell D, Barbour B, Szatkowski M (1993) Nonvesicular release of neurotransmitter. Neuron 11:401-407.

Belachew S, Gallo V (2004) Synaptic and extrasynaptic neurotransmitter receptors in glial precursors' quest for identity. Glia 48:185-196.

Butt AM, Ransom BR (1993) Morphology of astrocytes and oligodendrocytes during development in the intact rat optic nerve. J Comp Neurol 338:141-158.

Back, S.A. et al. (2006) Hypoxia-ischemia preferentially triggers glutamate depletion from oligodendroglia and axons in perinatal cerebral white matter. J Cereb Blood Flow Metab (Epub ahead of print).

Chiu SY, Kriegler S (1994) Neurotransmitter-mediated signaling between axons and glial cells. Glia 11:191-200.

Choi I, Chiu SY (1997) Expression of high-affinity neuronal and glial glutamate transporters in the rat optic nerve. Glia 20:184-192.

Danbolt NC (2001) Glutamate uptake. Prog Neurobiol 65:1-105.

Danbolt NC, Storm-Mathisen J, Kanner BI (1992) An [Na++ K+]coupled L-glutamate transporter purified from rat brain is located in glial cell processes. Neuroscience 51:295-310.

Dewar D, Underhill SM, Goldberg MP (2003) Oligodendrocytes and ischemic brain injury. J Cereb Blood Flow Metab 23:263-274.

Domercq M, Matute C (1999) Expression of glutamate transporters in the adult bovine corpus callosum. Brain Res Mol Brain Res 67:296-302.

Domercq M, Sanchez-Gomez MV, Areso P, Matute C (1999) Expression of glutamate transporters in rat optic nerve oligodendrocytes. Eur J Neurosci 11:2226-2236.

Domercq M, Etxebarria E, Perez-Samartin A, Matute C (2005) Excitotoxic oligodendrocyte death and axonal damage induced by glutamate transporter inhibition. Glia.

Furuta A, Rothstein JD, Martin LJ (1997a) Glutamate transporter protein subtypes are expressed differentially during rat CNS development. J Neurosci 17:8363-8375.

Furuta A, Martin LJ, Lin CL, Dykes-Hoberg M, Rothstein JD (1997b) Cellular and synaptic localization of the neuronal glutamate transporters excitatory amino acid transporter 3 and 4. Neuroscience 81:1031-1042.

Gegelashvili G, Schousboe A (1998) Cellular distribution and kinetic properties of highaffinity glutamate transporters. Brain Res Bull 45:233-238.

Gundersen V, Danbolt NC, Ottersen OP, Storm-Mathisen J (1993) Demonstration of glutamate/aspartate uptake activity in nerve endings by use of antibodies recognizing exogenous D-aspartate. Neuroscience 57:97-111.

Gundersen V, Shupliakov O, Brodin L, Ottersen OP, Storm-Mathisen J (1995) Quantification of excitatory amino acid uptake at intact glutamatergic synapses by immunocytochemistry of exogenous D-aspartate. J Neurosci 15:4417-4428. 
Itoh T, Beesley J, Itoh A, Cohen AS, Kavanaugh B, Coulter DA, Grinspan JB, Pleasure D (2002) AMPA glutamate receptor-mediated calcium signaling is transiently enhanced during development of oligodendrocytes. J Neurochem 81:390-402.

Káradóttir R, Cavelier P, Bergersen LH, Attwell D (2005) NMDA receptors in oligodendrocyte physiology and pathology. Nature In Press.

Kinney HC, Back SA (1998) Human oligodendroglial development: relationship to periventricular leukomalacia. Semin Pediatr Neurol 5:180-189.

Kugler P, Beyer A (2003) Expression of glutamate transporters in human and rat retina and rat optic nerve. Histochem Cell Biol 120:199-212.

Leppanen L, Stys PK (1997) Ion transport and membrane potential in CNS myelinated axons I. Normoxic conditions. J Neurophysiol 78:2086-2094.

Li S, Mealing GA, Morley P, Stys PK (1999) Novel injury mechanism in anoxia and trauma of spinal cord white matter: glutamate release via reverse $\mathrm{Na}+$-dependent glutamate transport. J Neurosci 19:RC16.

LoPachin RM, Jr., Stys PK (1995) Elemental composition and water content of rat optic nerve myelinated axons and glial cells: effects of in vitro anoxia and reoxygenation. J Neurosci 15:6735-6746.

Matute C, Alberdi E, Domercq M, Perez-Cerda F, Perez-Samartin A, Sanchez-Gomez MV (2001) The link between excitotoxic oligodendroglial death and demyelinating diseases. Trends Neurosci 24:224-230.

Medrano S, Steward O (2001) Differential mRNA localization in astroglial cells in culture. J Comp Neurol 430:56-71.

Micu I, Jiang Q, Coderre E, Ridsdale A, Zhang L, Woulfe J, Yin X, Trapp BD, McRory JE, Rehak R, Zamponi GW, Wang W, Stys PK (2006) NMDA receptors mediate calcium accumulation in myelin during chemical ischaemia. Nature 439:988-992.

Oka A, Belliveau MJ, Rosenberg PA, Volpe JJ (1993) Vulnerability of oligodendroglia to glutamate: pharmacology, mechanisms, and prevention. J Neurosci 13:1441-1453.

Pantoni L, Garcia JH, Gutierrez JA (1996) Cerebral white matter is highly vulnerable to ischemia. Stroke 27:1641-1646; discussion 1647.

Rothstein JD, Martin L, Levey AI, Dykes-Hoberg M, Jin L, Wu D, Nash N, Kuncl RW (1994) Localization of neuronal and glial glutamate transporters. Neuron 13:713725.

Salter MG, Fern R (2005) NMDA Receptors are Expressed in Developing Oligodendrocyte Processes and Mediate Injury. Nature 438:1167-1171.

Shimada N, Graf R, Rosner G, Heiss WD (1993) Ischemia-induced accumulation of extracellular amino acids in cerebral cortex, white matter, and cerebrospinal fluid. J Neurochem 60:66-71.

Stys PK (2004) White matter injury mechanisms. Curr Mol Med 4:113-130.

Tekkok SB, Goldberg MP (2001) Ampa/kainate receptor activation mediates hypoxic oligodendrocyte death and axonal injury in cerebral white matter. J Neurosci 21:4237-4248.

Thomas R, Salter MG, Wilke S, Husen A, Allcock N, Nivison M, Nnoli AN, Fern R (2004) Acute ischemic injury of astrocytes is mediated by Na-K-Cl cotransport and not $\mathrm{Ca} 2+$ influx at a key point in white matter development. J Neuropathol Exp Neurol 63.:856-871. 
Vannucci RC, Vannucci SJ (2000) Glucose metabolism in the developing brain. Semin Perinatol 24:107-115.

Volpe JJ (2001) Neurobiology of periventricular leukomalacia in the premature infant. Pediatr Res 50:553-562.

Volpe JJ (2003) Cerebral white matter injury of the premature infant-more common than you think. Pediatrics 112:176-180.

Wilke S, Salter MG, Thomas R, Allcock N, Fern R (2004) Mechanism of acute ischemic injury of oligodendroglia in early myelinating white matter: the importance of astrocyte injury and glutamate release. J Neurol Exp Neuropath 63:872-881.

Yamada K, Watanabe M, Shibata T, Nagashima M, Tanaka K, Inoue Y (1998) Glutamate transporter GLT-1 is transiently localized on growing axons of the mouse spinal cord before establishing astrocytic expression. J Neurosci 18:5706-5713.

Yuan X, Chittajallu R, Belachew S, Anderson S, McBain CJ, Gallo V (2002) Expression of the green fluorescent protein in the oligodendrocyte lineage: a transgenic mouse for developmental and physiological studies. J Neurosci Res 70:529-545. 

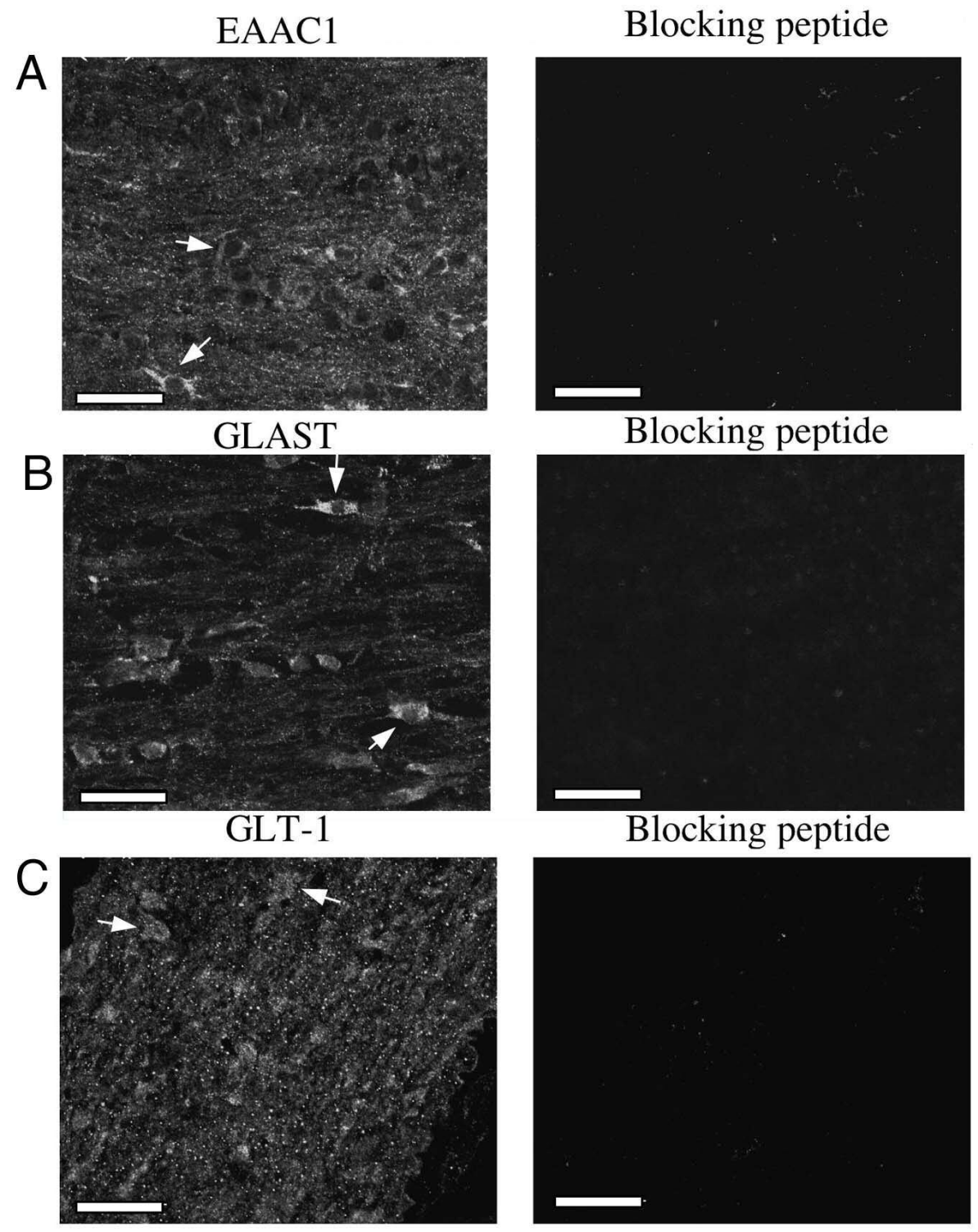

Blocking peptide

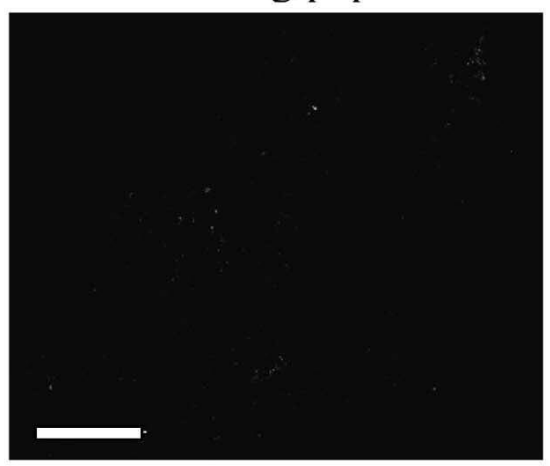

Figure 1 Glutamate transporter expression in neonatal white matter. EAAC1 (A), GLAST (B) and GLT-1 (C) expression has both a somata pattern of expression (arrows) and nonsomata pattern of expression in neonatal optic nerve. Antibody staining is not seen when the antibodies are pre-incubated in the relevant blocking peptides (right side). Scale bar $=10 \mu \mathrm{m}$. 


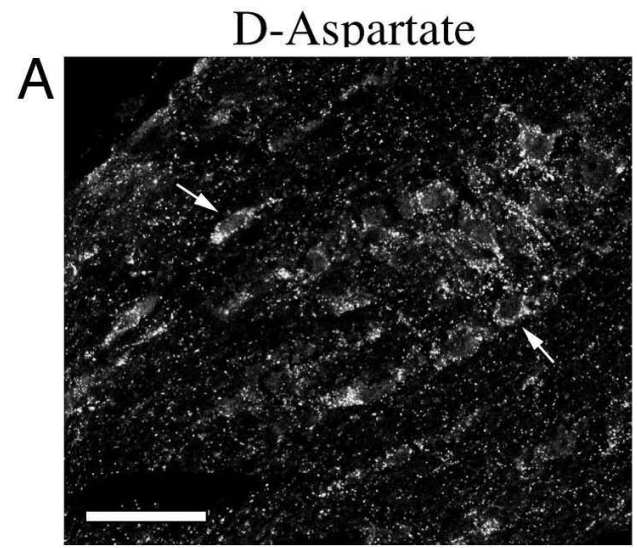

Zero-Na

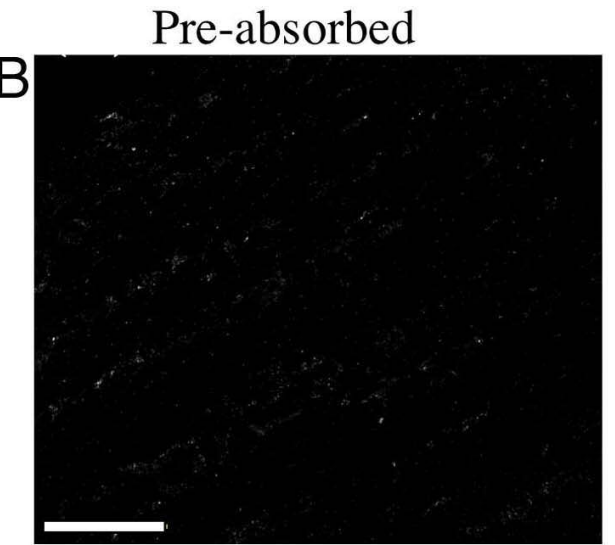

TBAO

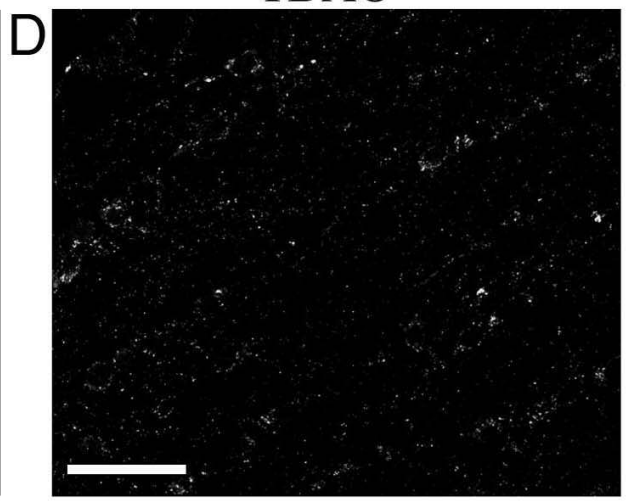

Figure 2 D-aspartate uptake into neonatal white matter is via functional glutamate transport. A: Antibody staining for D-aspartate showing uptake into somata (arrows) and non-somata areas of the pre-incubated optic nerve. B: Pre-absorbing the antibody with Daspartate prior to incubation blocked D-aspartate staining. C-D: D-aspartate uptake was blocked by removing extracellular $\mathrm{Na}+(\mathrm{C})$ or by TBOA (D), a specific glutamate transport blocker. Scale bar $=10 \mu \mathrm{m}$. 


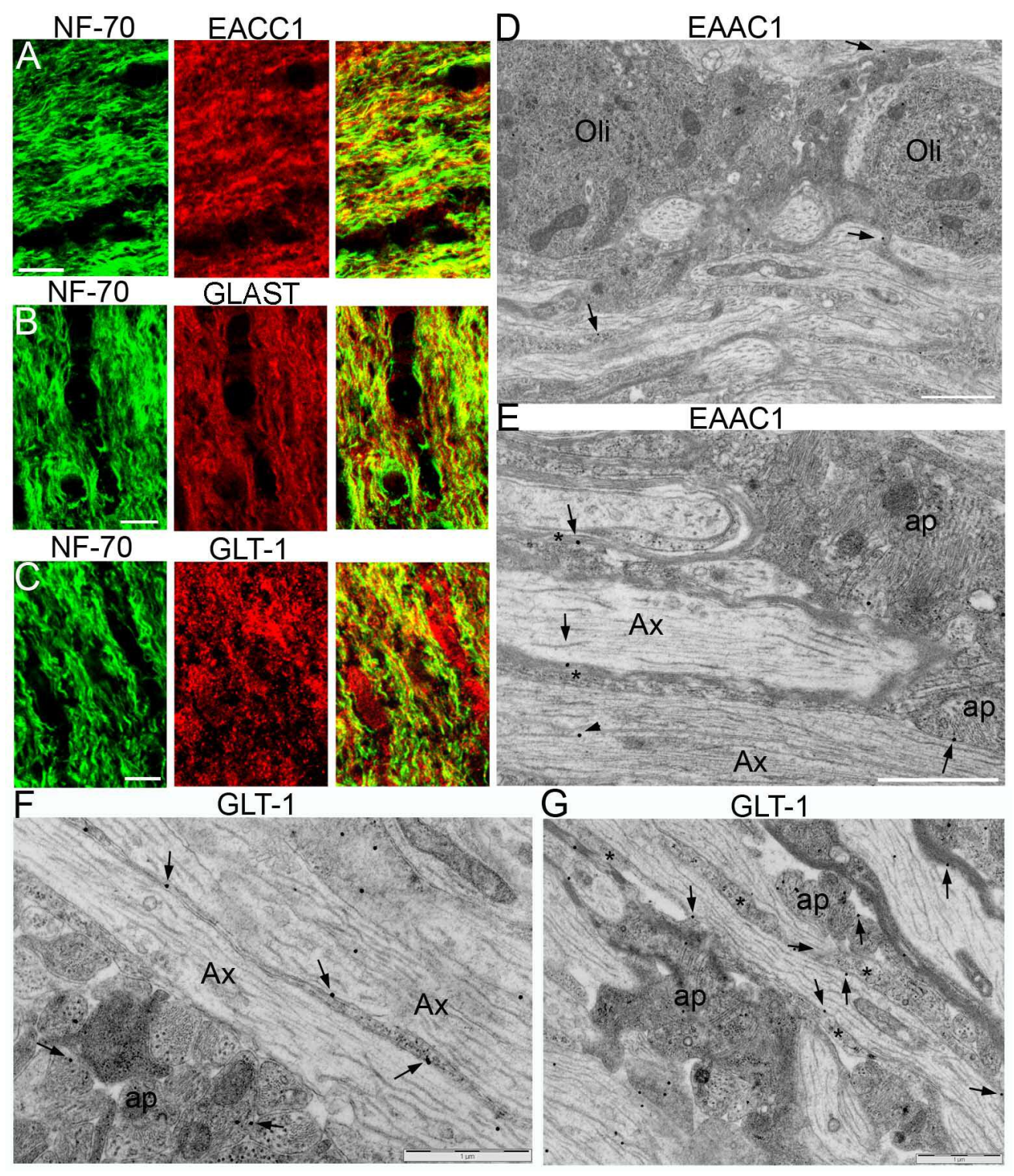

Figure 3 Glutamate transporter expression in neonatal mouse axons. A-C: Axons are costained with NF-70 (left panels, green) and for either; EAAC1 (A, middle panel, red), GLAST (B, middle panel, red) or GLT-1 (C, middle panel, red). The images are overlaid on the panels to the right, showing what appears to be significant co-localization of all three transporters in axons (yellow). D, E: Immuno-gold reactivity for EAAC1. D: Gold particles are found throughout the cytoplasm and in particular in the cell membrane of oligodendrocytes (arrows). E: Staining is evident in oligodendrocyte processes (asterisks + arrows) that are closely apposed to axons (Ax). Light staining was found in the axoplasm of axons (arrow head), but not in the axolemma. Note on the right a rare gold particle in the cell membrane of an astrocyte process (ap) aligned next to an axon (arrow). F, G: Immuno-gold reactivity for GLT-1. Note the heavy gold-particle labelling within the axolemma in both panels, in addition to staining within the axoplasm and within the membrane of astrocyte processes (ap). Scale bar $=10 \mu \mathrm{m}$ in A-C and $1 \mu \mathrm{m}$ in D-G. The cell types are identified using standard criteria for this preparation (Thomas et 
al., 2004).

al., 2004). (1)

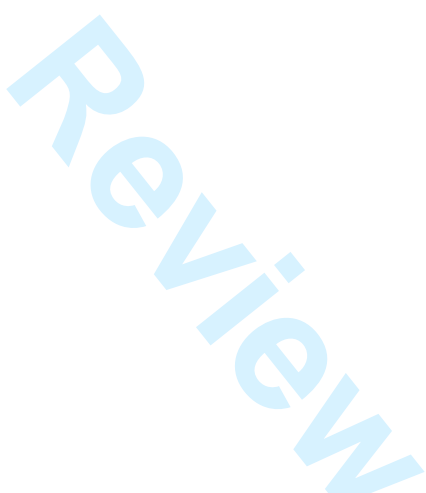

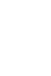

(20.

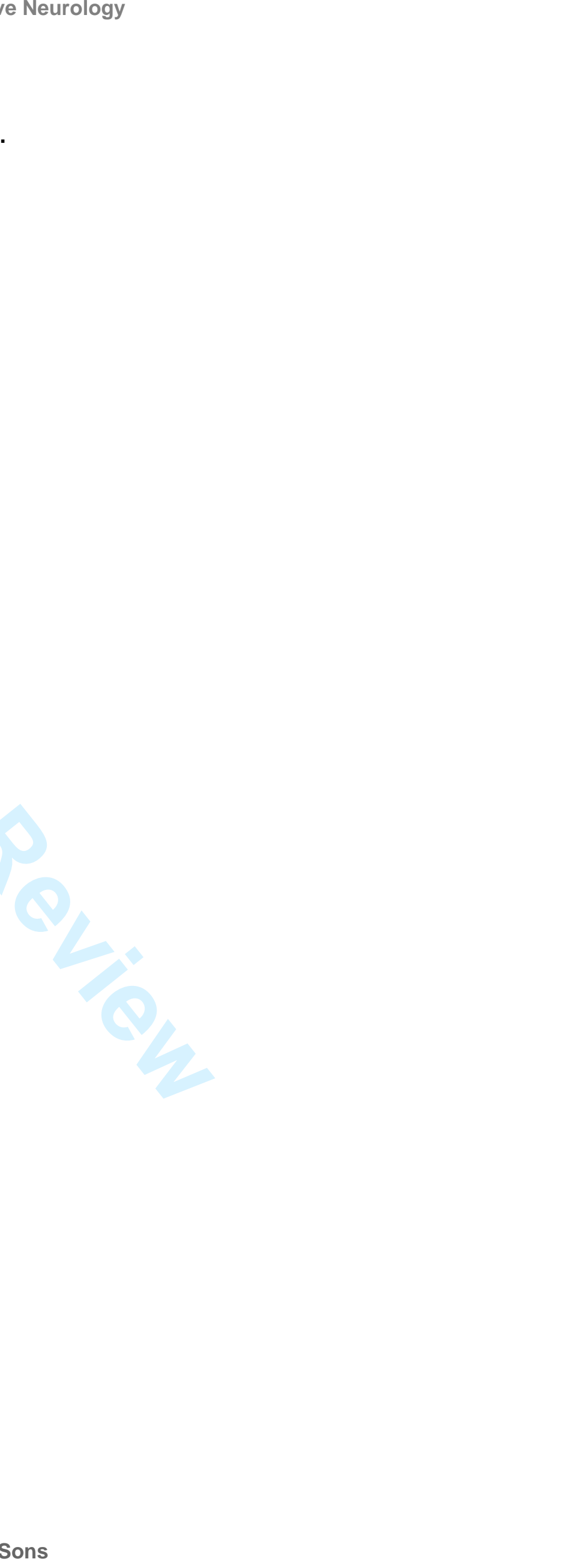



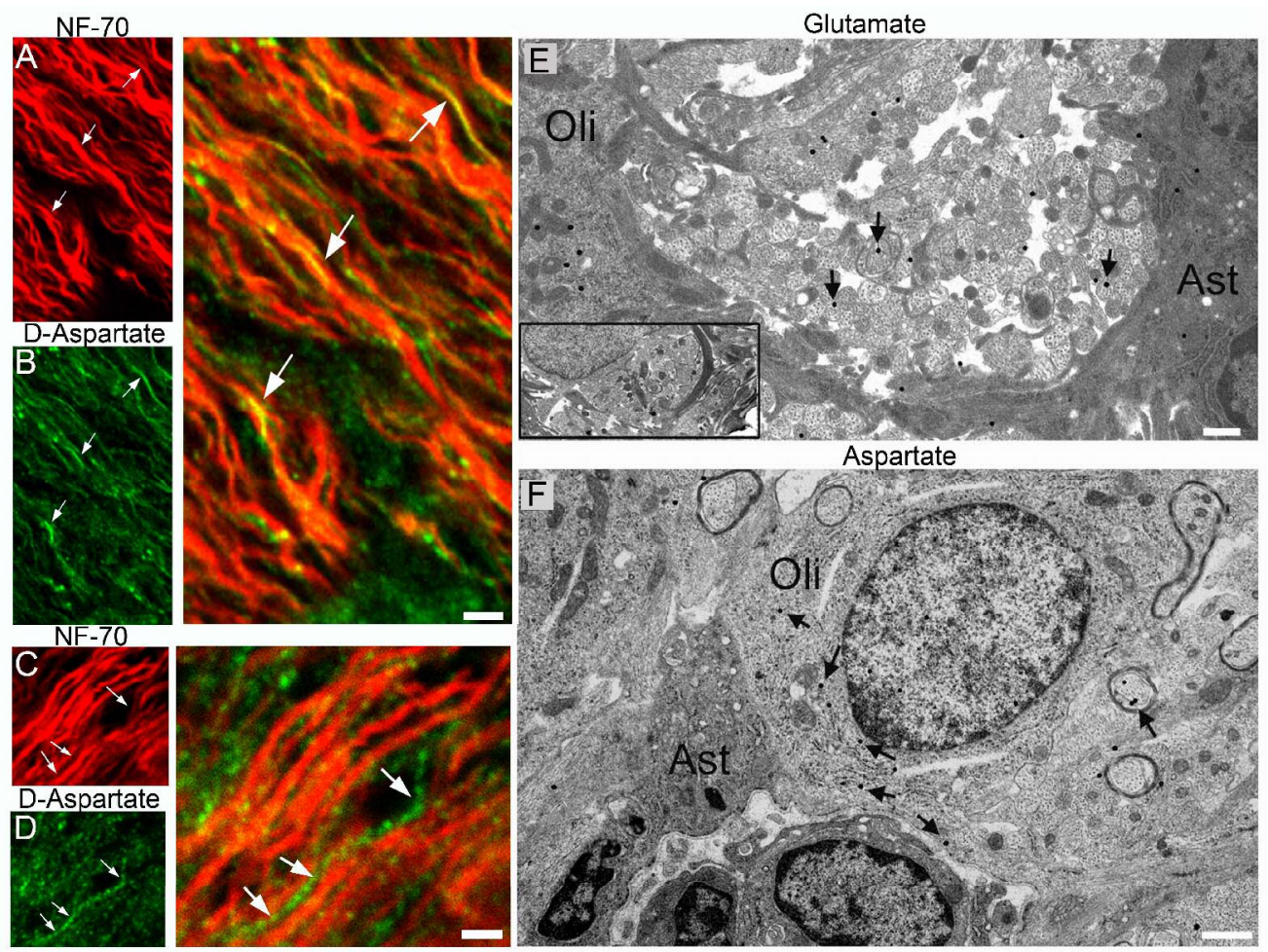

Figure 4 Glutamate and D-aspartate staining in neonatal mouse optic nerve. A: NF-70 staining of axons. B: D-aspartate staining of the same section, overlaid to the right. Note the presence of D-aspartate staining within axons (arrows). C: NF-70 staining of axons. D: D-aspartate staining of the same section, overlaid to the right. Note the D-aspartate staining within a NF-70 (-) glial process running parallel to the NF-70 (+) axons. E:

Immuno-gold localization of glutamate reactivity showing gold particles within an

oligodendrocyte (Oli), an astrocyte (Ast) and within axons (arrows). The box shows absence of staining when the antibody was pre-incubated with glutamate. F: D-aspartate reactivity in pre-incubated optic nerve. Note the gold particles within an oligodendrocyte and within axons (arrows) and the absence of staining within the astrocyte. Scale bars = $10 \mu \mathrm{m}$ in $A-D$ and $1 \mu \mathrm{m}$ in $E-F$. 

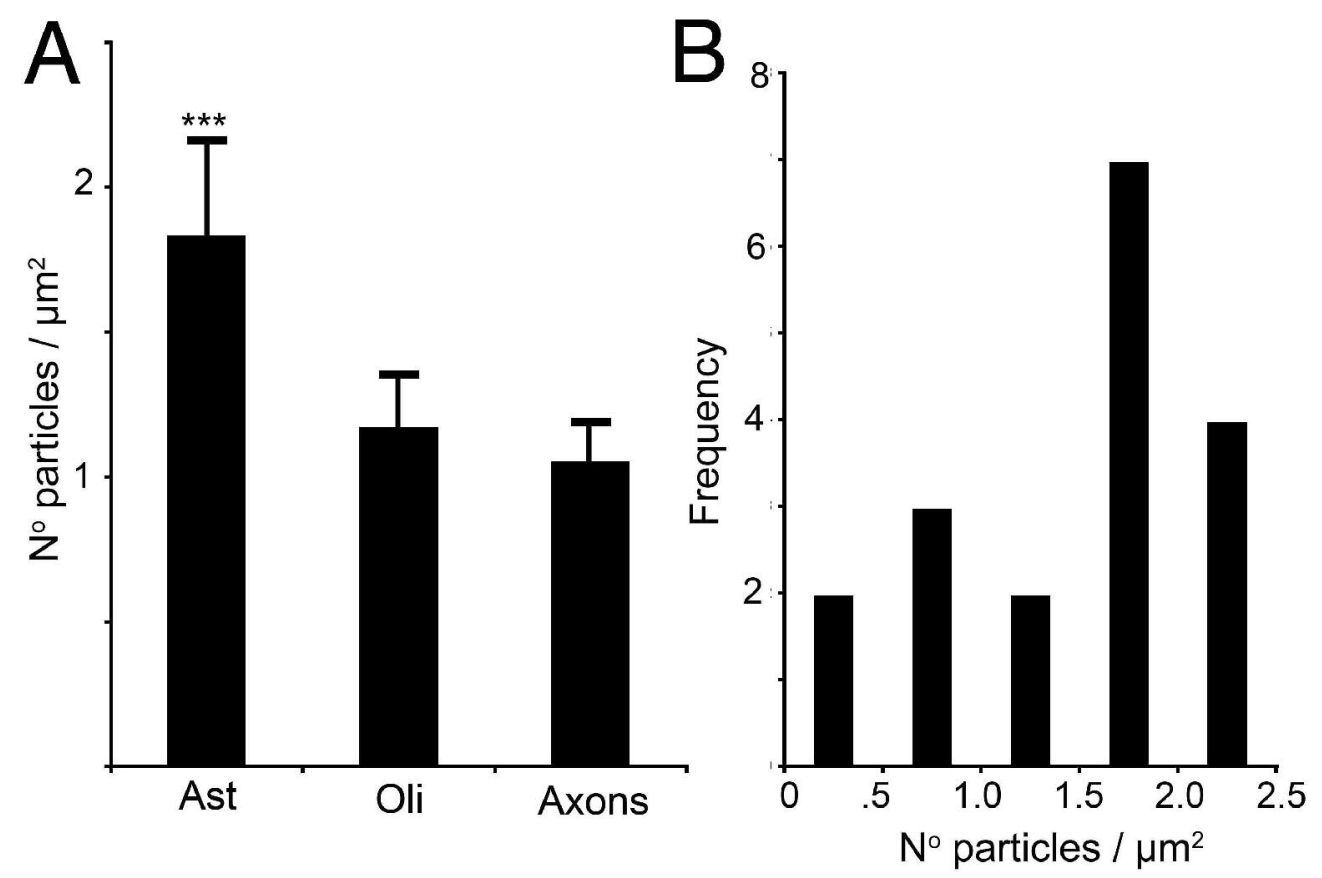

Figure 5 Blinded counting of D-aspartate gold particles. A: Astrocytes accumulate significantly more $D$-aspartate than either axons or oligodendrocytes within the optic nerve B: The population distribution of gold-particle density within astrocytes was highly variable, with a population of astrocytes that accumulated little $D$-aspartate. Frequency refers to the number of astrocytes which fall within a given bin. $* * *=\mathbf{P}>0.001$. 


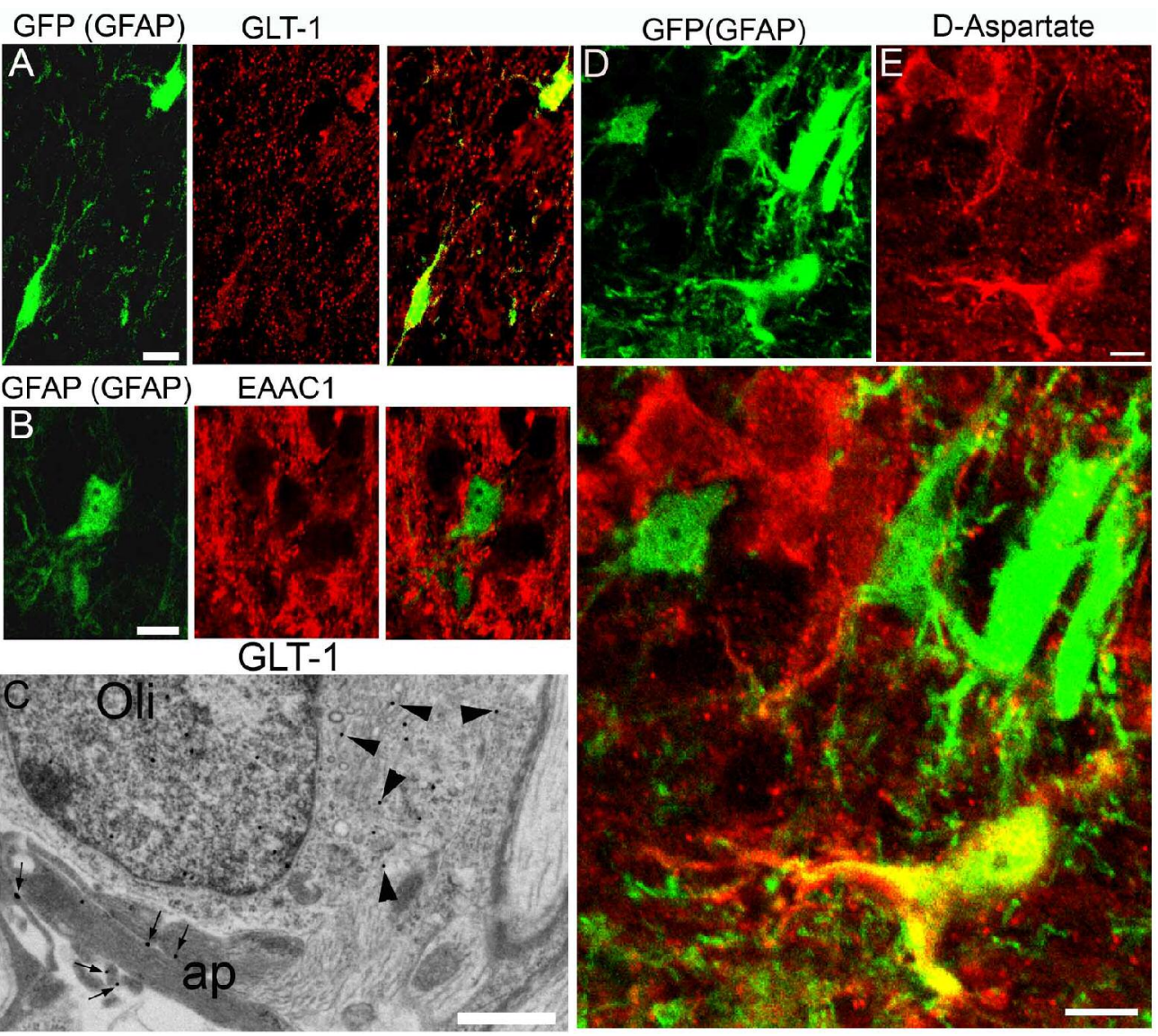

Figure 6 Glutamate transporter expression in neonatal white matter astrocytes. A, B: GFP expression in GFAP-GFP astrocytes (green, left) and labelling for either GLT-1 (A, red, middle) and EAAC1 (B, red, middle). The images are overlaid on the right, showing significant expression of GLT-1 in astrocytes with no co-expression of EAAC1. C: Immunogold labelling for GLT-1 showing expression in the membrane of astrocyte processes ("ap", arrows) and staining only in the cytoplasm of a neighbouring oligodendrocyte ("Oli", arrow-heads). D: GFP expression in astrocytes. E: Co-staining for D-aspartate in the same pre-incubated nerve. The images are overlaid below, showing D-aspartate uptake into one of the astrocytes (yellow) with several D-aspartate $(-)$ astrocytes present. Note the presence of two D-aspartate $(+)$ somata (left top corner) that are GFP $(-)$, presumably oligodendrocytes. Scale bar = $10 \mu \mathrm{m}$ except in "C" where it is $1 \mu \mathrm{m}$. 

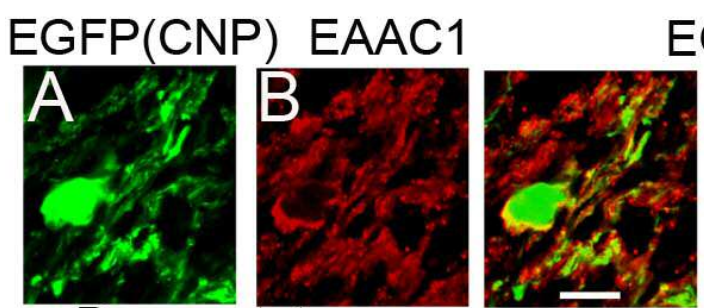

EGFP(CNP) GLT-1

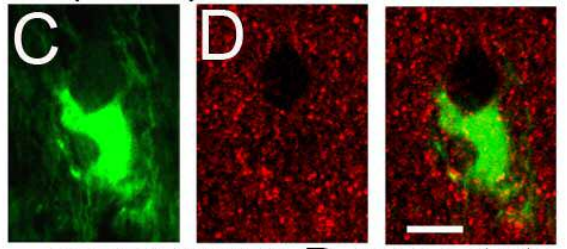

D-aspartate

CNPase
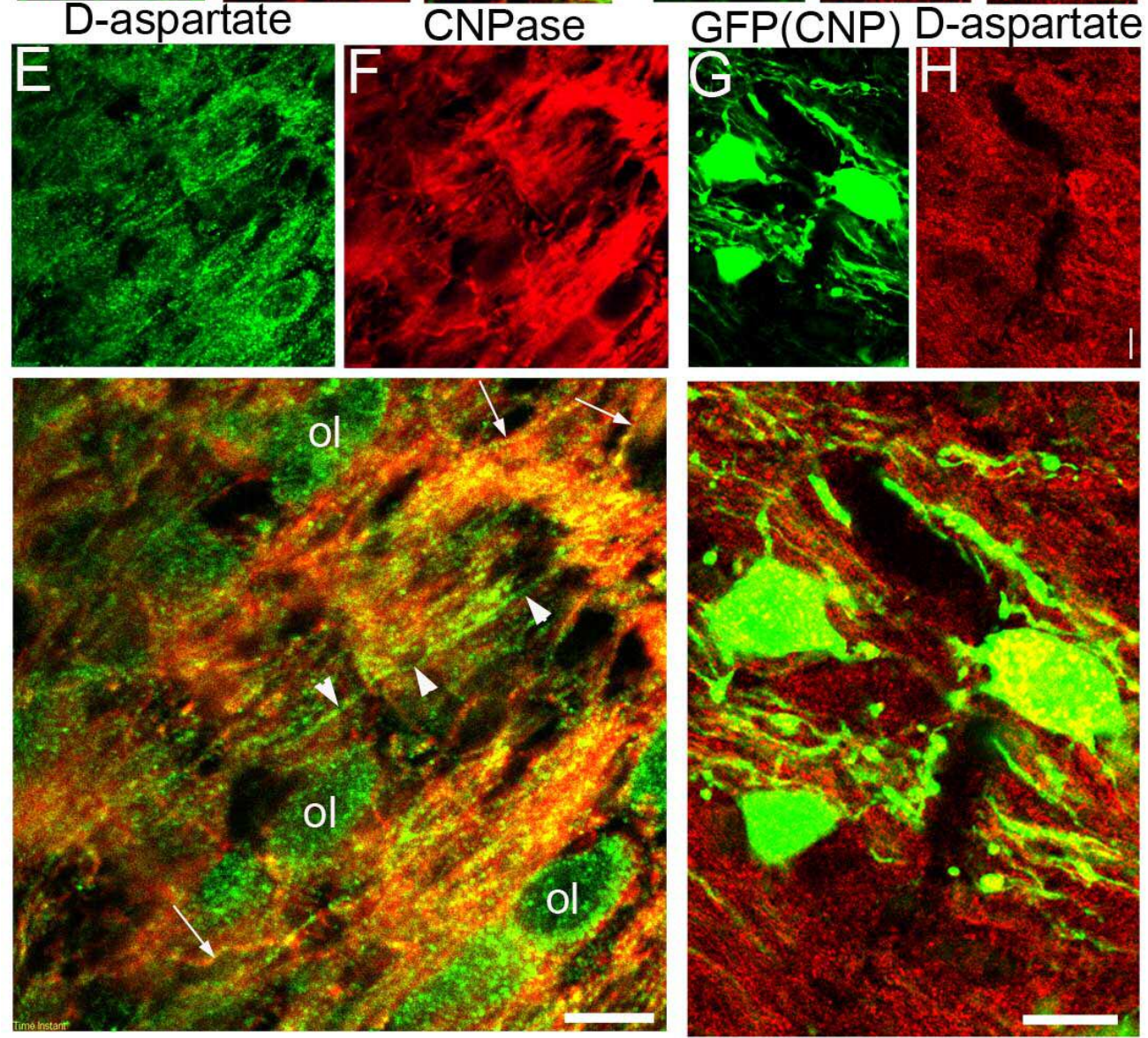

Figure 7 Glutamate transporter expression in neonatal white matter oligodendrocytes. A: GFP expression in a CNP-EGFP oligodendrocyte showing soma and processes. B: EAAC1 reactivity of the same section. Overlay on the right, showing EAAC1 expression on the soma and processes. C: A single CNP-EGFP (+) oligodendrocyte. D: GLT-1 reactivity of the same section. Overlay on the right, showing diffuse GLT staining in the cell. E: Daspartate staining of a wild-type, D-aspartate pre-incubated, nerve. F: CNPase staining of the same section. Overlay below, showing clear D-aspartate staining in the CNPase (+) oligodendrocyte processes (small arrows) as well as within CNPase (-) axon-like structures (arrow heads). G: Three EGFP (+) oligodendrocytes in a CNP-EGFP nerve. H: Daspartate staining of the same section. Overlay below, showing D-aspartate staining within somata and proximal processes of oligodendrocytes. Scale bars $=10 \mu \mathrm{m}$. 


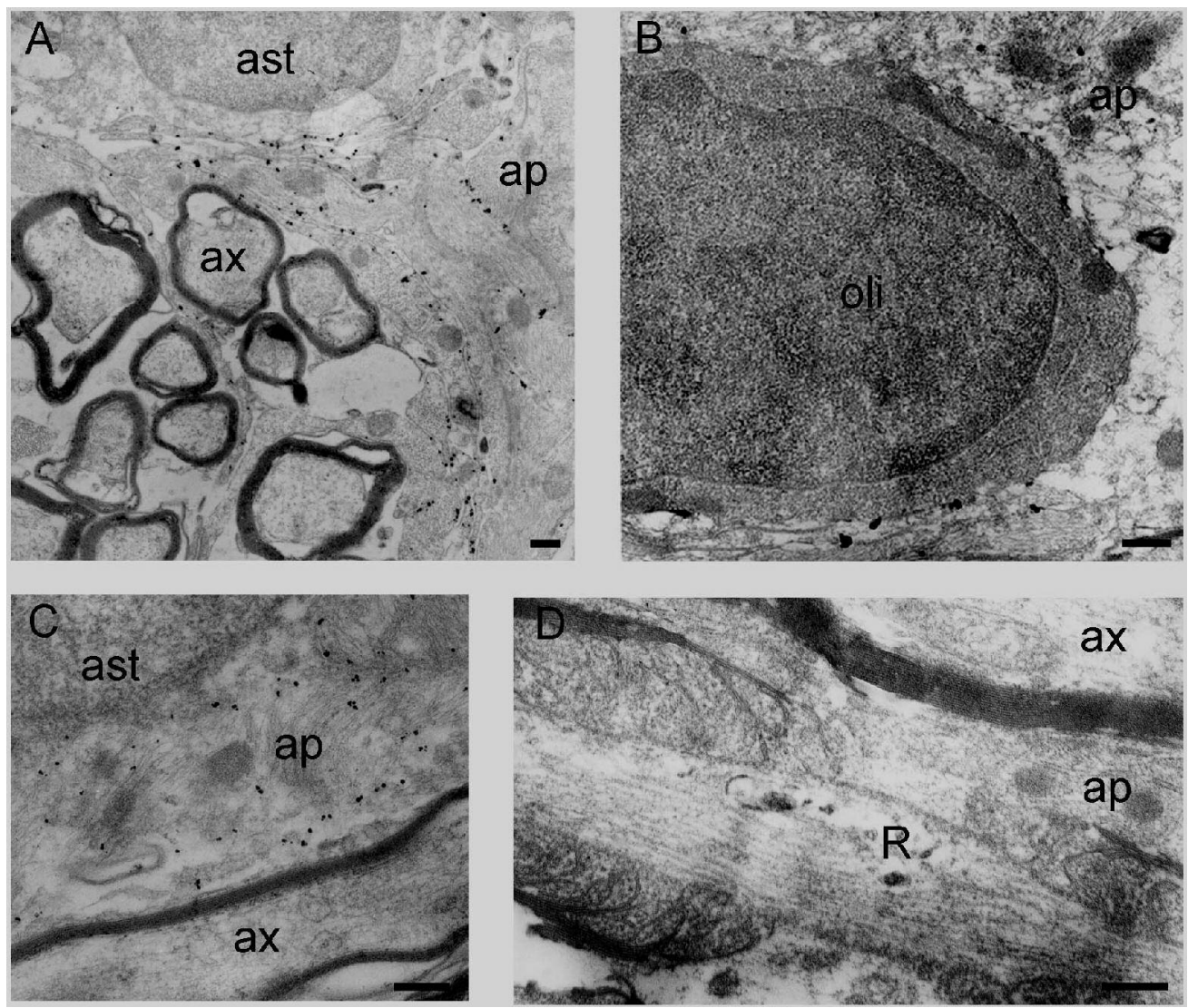

Figure 8 Ultrastructural localization of GLT-1 in adult rat optic nerve. A: Astrocyte processes (ap) with intense GLT-1 immuno-labelling surrounding a group of axons (ax) all devoid of reactivity. B, C: Transverse and longitudinal sections showing that GLT-1 is present at high levels in astrocyte processes (ap) and to a lesser extent in oligodendrocytes (oli, arrows). D: No expression of GLT-1 is observed in axons and at the nodes of Ranvier $(R)$. Scale bars $=300 \mathrm{~nm}$. ast = astrocytic nucleus 

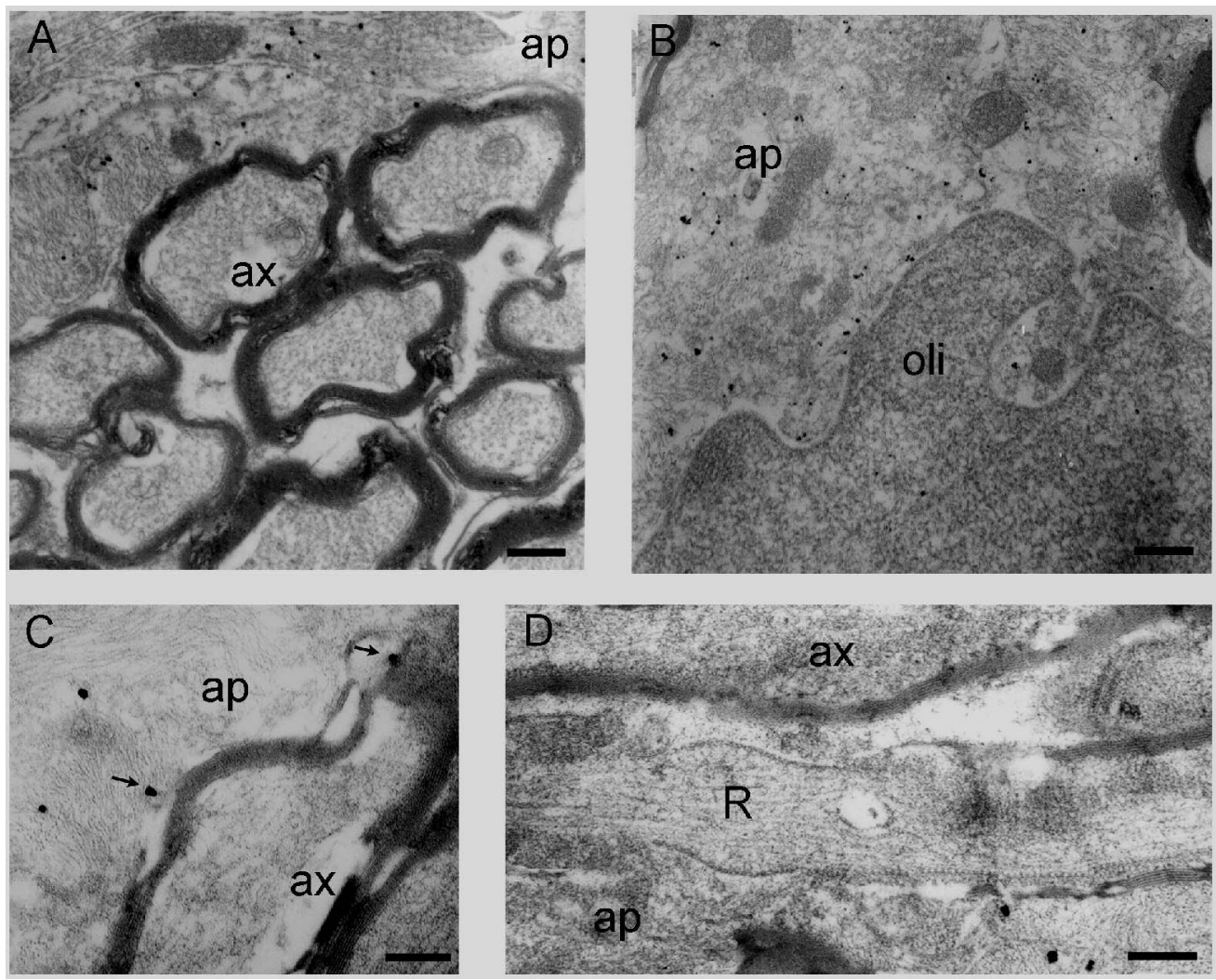

Figure 9 Ultrastructural localization of GLAST in adult rat optic nerve. A, C: Transverse and longitudinal sections showing GLAST localization in astrocyte processes (ap) that surround some axons (ax) free of labelling and also in myelin sheaths (arrows). Note that immuno-particles are present in plasma membranes as well as in the cytoplasm of astrocytes. B: Expression of GLAST in astrocytes and to a lesser extent in oligodendrocytes. D: Axons (ax) and the nodes of Ranvier (R) are unlabelled. Scale bars = $300 \mathrm{~nm}$. 

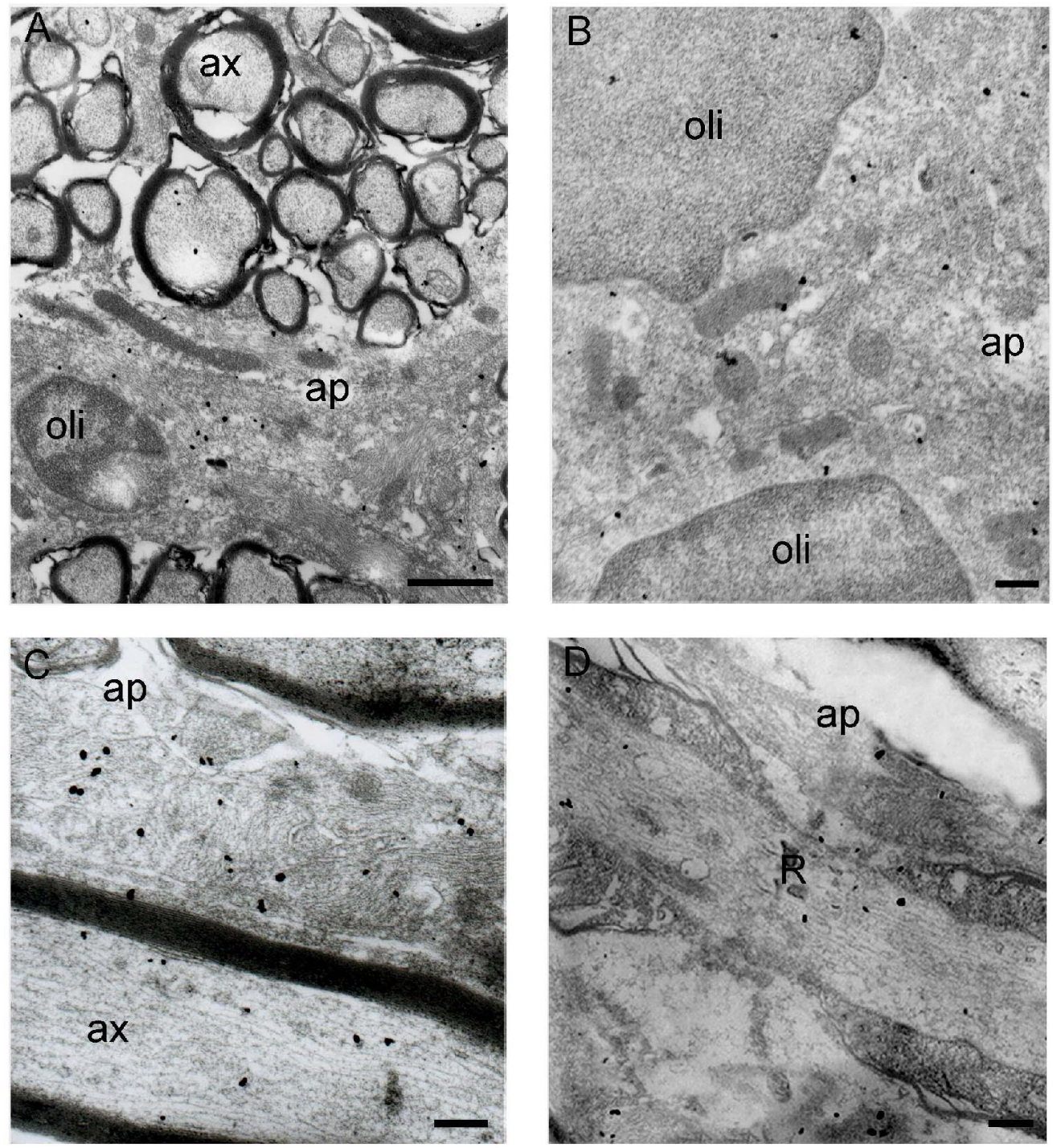

Figure 10 Ultrastructural localization of EAAC1 in adult rat optic nerve. A, C: Transverse and longitudinal sections showing EAAC1 expression in both axons (ax) and astrocyte processes (ap). Note that immuno-labelling frequently appears in the cytoplasm. B: EAAC1 is also found in oligodendrocytes (oli) as well as in astrocyte processes (ap) in their vicinity. D: Note that reactivity is particularly abundant at the nodes of Ranvier $(R)$. Scale bars $=300 \mathrm{~nm}$. 

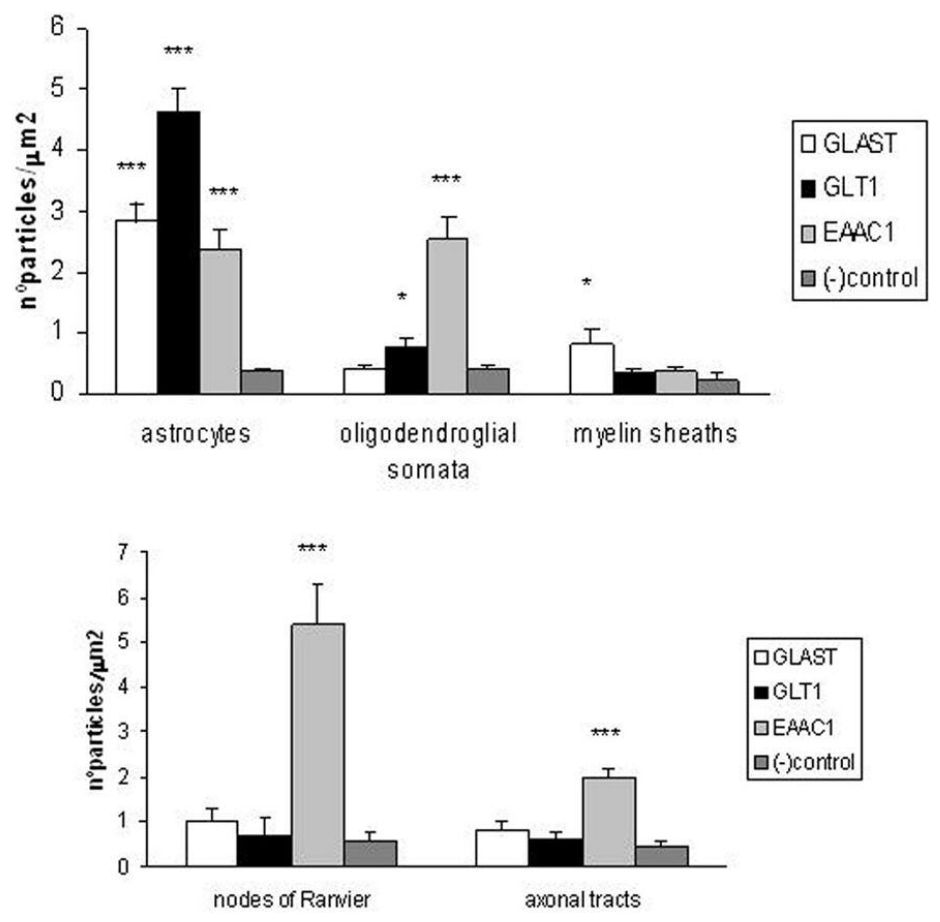

Figure 11 Quantitative analysis of the distribution of glutamate transporters in adult optic nerve. All transporters are abundantly expressed in astrocytes. Oligodendrocytes express lower levels of GLT-1 and EAAC1, and myelin has GLAST. Axons and nodes of Ranvier have moderate and high levels of EAAC1, respectively. * $\mathbf{p}<0.05, * * * \mathbf{p}<0.001$, as compared to negative control sections not incubated with primary antibody. 